Physical sciences / Energy science and technology / Energy storage / Batteries

[URI /639/4077/4079/891]

\title{
Batteries: Beyond intercalation and conversion
}

Positive electrode materials for lithium-ion batteries feature host structure to reversibly insert and conduct lithium element. Now, electrochemically activated transition metal oxide-lithium fluoride composite materials are shown to have potential for high-capacity positive electrodes

Alexis Grimaud

Since the introduction of lithium-ion batteries (LIB) in the early 1990's, this technology has deeply modified our world. Indeed, energy-dense rechargeable batteries spurred the spread of portable electronics, enabled the realization of electric vehicles with a reasonable driving range and are currently envisioned as part of the solution for energy grid storage. ${ }^{1}$ The success of such disruptive technology was made possible by the development of lithiumcontaining transition metal oxides such as $\mathrm{LiCoO}_{2}$ for the positive electrode, thereby removing the need for a metallic lithium source at the negative electrode. Relaxing this constraint unleashed creativity to propose a variety of new negative electrodes. Subsequently, different classes of materials began to emerge, such as insertion compounds (graphite or early transition metal oxides), elements forming alloys with lithium (e.g. silicon) or conversion materials (nano-size transition metal oxides). While research on negative electrodes has illustrated this innovative spirit in electrode design, insertion materials are still acclaimed as the positive electrodes of choice. Among the different classes of intercalation materials that exist, with the predominant ones being the layered oxides and the polyanionic compounds, all currently rely on the redox chemistry of active transition metals forming a host structure for lithium to reversibly intercalate upon cycling. ${ }^{2}$ The weight of the host structure however limits the capacity of insertion electrodes, which in turn limits the performances of LIBs. Although conversion cathodes, using metal fluorides, were previously studied and demonstrate good cycling performances at a potential around $3 \mathrm{~V}^{3}$ an additional chemical lithiation step was so far required in order to use them as positive electrodes. ${ }^{4}$ Writing in Nature Energy, Kisuk Kang and coworkers in Korea now address these limitations using nano-sized transition metal oxides (MO) intimately blended with LiF particles, the latter of which act as the lithium source. ${ }^{5}$

The researchers showed that upon an initial charge, the surface of the so-formed MnO-LiF composite electrodes is activated at high potentials $(\sim 4.5 \mathrm{~V})$, leading to the formation of a new active phase (Fig. 1) that can be subsequently reversibly cycled. To demonstrate the surface activation process taking place at high potentials, the team utilized in situ X-ray absorption spectroscopy to track the reversible change of manganese oxidation state during cycling. With the aid of surface and bulk sensitive techniques, they found that upon charging the oxidation process mostly occurs on the surface of the $\mathrm{MnO}$ particles. Using electron 
energy loss spectroscopy and scanning transmission electron microscopy, they further observed that the oxidation is correlated with the presence of fluorine at the surface of the particles after charging. Therefore, the activation process was assigned to the formation of an oxyfluoride MnO-F-like shell (Fig. 1). Combined with other characterization techniques, Kang and coworkers demonstrated that LiF particles initially contained in the pristine $\mathrm{MnO}$ LiF composites decomposed during the initial charge and that the surface of the metal oxide particles undergoes a phase transformation from a rock salt to a spinel-like structure (dubbed as diffusionless transformation) ; upon further cycling lithium is then reversibly exchanged. As shown in Fig. 1, this diffusionless surface transformation largely differs from the intercalation process controlled by the bulk diffusion and which is commonly observed in conventional LIB cathodes, explaining the good rate capabilities measured for these electrodes. Overall, almost one lithium ion is exchanged during this conversion reaction and the initial capacity approaches the theoretical one of $263 \mathrm{mAh} / \mathrm{g}$. These metrics make this type of positive electrode competitive with the current state of the art lithium rich layered compounds, which deliver a reversible capacity of approximately $260 \mathrm{mAh} / \mathrm{g}$.

Kang and coworkers also established that this reaction is not limited to MnO but can be extended to iron- and cobalt- based nanocomposite electrodes. They emphasize the flexibility of such surface-controlled reactions by demonstrating that the capacity can be tuned by simply controlling the size of $\mathrm{Mn}_{3} \mathrm{O}_{4}$ particles, for which almost two electrons can be exchanged during the cycling.

This work represents a major step forward in the design of new positive electrodes; however many questions remain to be solved before the practical use of such composite conversion electrodes can be envisioned. In particular, one can anticipate that challenges identical to those hampering the development of nano-size transition metal oxides as conversion anode materials ${ }^{6}$ will be encountered for this new class of positive electrodes. Indeed, increasing the exposed surface in contact to the electrolyte and employing high potentials $(>4.5 \mathrm{~V})$ to activate the surface of these particles will lead to challenges related to the electrolyte stability. Hence, side reactions were recently reported in a separate study when using similar materials that employed $\mathrm{LiPF}_{6}$ salt, instead of $\mathrm{LiF}$, as lithium and fluorine source to electrochemically prepare the oxyfluoride active phase. ${ }^{7}$ Even though oxidizing the $\mathrm{LiPF}_{6}$ salt could be seen as promising, it is accompanied by drastic solvent oxidation at high potential as detected by mass spectroscopy. Moreover, poor round trip efficiencies associated with large polarization upon charge and discharge are observed even at low rates by Kang and coworkers, similarly to what has been previously observed for conversion materials used as negative electrodes. Knowing that this limitation related to the large polarization observed has never been tackled for conversion anode materials, one can legitimately wonder if the same will be true for positive electrodes. Finally, the need of an additional activation step can represent a major drawback for the applicability of such reactions.

Nevertheless, exciting opportunities could arise from this work to open up a new field for the development of positive electrodes for LIBs. It also offers a chance to better understand the electrochemical and chemical steps governing the conversion processes so as to design new reaction paths to reversibly exchange lithium at high potential, and potentially stabilize new metastable oxyfluoride phases. 
Alexis Grimaud is at Chimie du Solide et de l'Energie, UMR 8260, Collège de France, 75231 Paris Cedex 05, and Réseau sur le Stockage Electrochimique de l'Energie (RS2E), FR CNRS 3459, 80039 Amiens Cedex, France. Email: Alexis Grimaud <alexis.grimaud@college-de-france.fr>

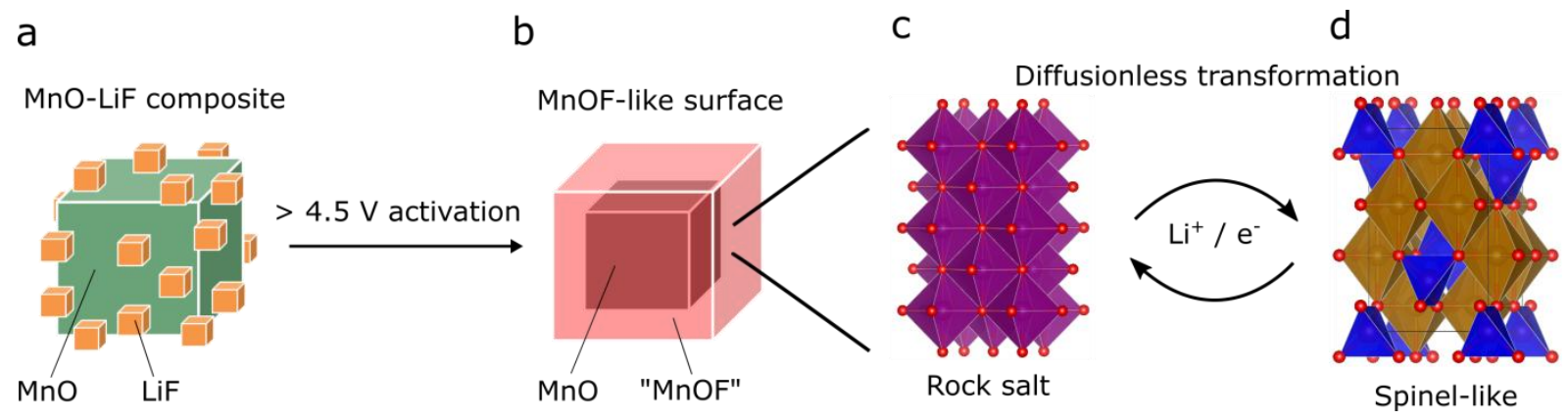

Figure 1 Surface activation of MnO-LiF composites The MnO-LiF composite material (a) is first formed by ball-milling. The composite is then oxidized at high potential $(>4.5 \mathrm{~V})$ during the first charge to form a MnOF-like surface (b) which possesses a rock-salt structure (c). The rock-salt surface is electrochemically active for the lithium insertion and can reversibly transforms through a diffusionless process into a spinel-like surface (d).

1. Gupta, S. Nature 526, S90-S91 (2015).

2. Grimaud, A., Hong, W. T., Shao-Horn, Y. \& Tarascon, J.-M. Nat. Mater. 15, 121-126 (2016).

3. Wu, F. \& Yushin, G. Energy Environ. Sci. (2017). doi:10.1039/C6EE02326F

4. Amatucci, G. G. et al. J. Fluor. Chem. 132, 1086-1094 (2011).

5. Jung, S.-K. et al. Nat. Energy (2016).

6. Cabana, J., Monconduit, L., Larcher, D. \& Palacín, M. R. Adv. Energy Mater. 22, E170-92 (2010).

7. Zhang, L., Chen, G., Berg, E. J. \& Tarascon, J.-M. Adv. Energy Mater. (2016). doi:10.1002/aenm201602200 